International Journal of Pure and Applied Mathematics

Volume 95 No. $4 \quad 2014,567-575$

ISSN: 1311-8080 (printed version); ISSN: 1314-3395 (on-line version)

url: http://www.ijpam.eu

doi: http://dx.doi.org/10.12732/ijpam.v95i4.9

ijpam.eu

\title{
DIRICHLET-LAURICELLA TYPE D DISTRIBUTION
}

\author{
Daya K. Nagar ${ }^{1}$, Armando Gómez ${ }^{2}$ \\ ${ }^{1,2}$ Instituto de Matemáticas \\ Universidad de Antioquia
}

Calle 67, No. 53-108, Medellín, COLOMBIA

\begin{abstract}
In this article, we define and study the Dirichlet-Lauricella type D distribution. This distribution is a generalization of the Dirichlet type 1 distribution.
\end{abstract}

AMS Subject Classification: $62 \mathrm{H} 99$

Key Words: Appell hypergeometric function, Dirichlet distribution, hypergeometric function, Lauricella function

\section{Introduction}

The random variables $U_{1}, \ldots, U_{n}$ are said to have a Dirichlet type 1 distribution with parameters $a_{1}, \ldots, a_{n} ; a_{n+1}$, denoted by $\left(U_{1}, \ldots, U_{n}\right) \sim \mathrm{D} 1\left(a_{1}, \ldots, a_{n}\right.$; $\left.a_{n+1}\right)$, if their joint probability density function (p.d.f.) is given by

$$
\begin{array}{r}
\frac{\Gamma\left(\sum_{i=1}^{n+1} a_{i}\right)}{\prod_{i=1}^{n+1} \Gamma\left(a_{i}\right)} \prod_{i=1}^{n} u_{i}^{a_{i}-1}\left(1-\sum_{i=1}^{n} u_{i}\right)^{a_{n+1}-1}, \\
u_{i}>0, \quad i=1, \ldots, n, \quad \sum_{i=1}^{n} u_{i}<1 .
\end{array}
$$

The Dirichlet type 1 distribution is a multivariate generalization of the beta distribution. Dirichlet distributions are very often used as prior distributions in Bayesian statistics, and in fact the Dirichlet distribution is the conjugate prior

Received: June 9, 2014

(c) 2014 Academic Publications, Ltd.

$\S_{\text {Correspondence author }}$ url: www.acadpubl.eu 
of the categorical distribution and multinomial distribution. The Dirichlet type 1 distribution has been studied extensively, for example, see Kotz, Balakrishnan and Johnson [2], and Gupta and Nagar [3].

In this article, we give a generalization of the Dirichlet type 1 distribution. This generalization is based on the Lauricella type D hypergeometric function and thus will be called Dirichlet-Lauricella type D distribution. Recently, Nagar and Gómez [8] have proposed a generalization of Dirichlet type 1 distribution based on the Lauricell's type B hypergeometric function.

In Section 2, we give definition of Lauricella type D hypergeometric function. We define Dirichlet-Lauricella type D distribution in Section 3. Section 4, deals with several properties such as marginal densities and joint moment.

\section{Preliminaries}

The Pochhammer symbol $(a)_{n}$ is defined by $(a)_{n}=a(a+1) \cdots(a+n-1)=$ $(a)_{n-1}(a+n-1)$ for $n=1,2, \ldots$ and $(a)_{0}=1$. The Lauricella hypergeometric functions $F_{D}^{(n)}$ of several variables is defined as

$$
\begin{aligned}
& F_{D}^{(n)}\left(a, b_{1}, \ldots, b_{n} ; c ; z_{1}, \ldots, z_{n}\right) \\
& =\sum_{j_{1}, \ldots, j_{n}=0}^{\infty} \frac{(a)_{j_{1}+\cdots+j_{n}}\left(b_{1}\right)_{j_{1}} \cdots\left(b_{n}\right)_{j_{n}}}{(c)_{j_{1}+\cdots+j_{n}}} \frac{z_{1}^{j_{1}} \cdots z_{n}^{j_{n}}}{j_{1} ! \cdots j_{n} !}, \quad \max \left\{\left|z_{1}\right|, \ldots,\left|z_{n}\right|\right\}<1 .
\end{aligned}
$$

For $n=1$, the Lauricella hypergeometric functions $F_{D}^{(n)}$ reduces a Gauss hypergeometric function and for and $n=2$ it slides to an Appell hypergeometric function $F_{1}$.

The integral representations of $F_{D}^{(n)}$ is given by

$$
\begin{aligned}
& F_{D}^{(n)}\left(a, b_{1}, \ldots, b_{n} ; c ; z_{1}, \ldots, z_{m}\right) \\
& =\frac{\Gamma(c)}{\prod_{i=1}^{n} \Gamma\left(b_{i}\right) \Gamma\left(c-\sum_{i=1}^{n} b_{i}\right)} \\
& \quad \times \int_{\substack{u_{1}>0, \ldots, u_{n}>0 \\
1-\sum_{i=1}^{n} u_{i}>0}} \frac{\prod_{i=1}^{n} u_{i}^{b_{i}-1}\left(1-\sum_{i=1}^{n} u_{i}\right)^{c-\sum_{i=1}^{n} a_{i}-1}}{\left(1-\sum_{i=1}^{n} z_{i} u_{i}\right)^{a}} \prod_{i=1}^{n} \mathrm{~d} u_{i},
\end{aligned}
$$

where $\operatorname{Re}\left(b_{i}\right)>0, i=1, \ldots, n$ and $\operatorname{Re}\left(c-b_{1}-\cdots-b_{n}\right)>0$.

Another representation of $F_{D}^{(n)}$, in terms of a single integral, is given by

$$
F_{D}^{(n)}\left(a, b_{1}, \ldots, b_{n} ; c ; z_{1}, \ldots, z_{n}\right)=\frac{\Gamma(c)}{\Gamma(a) \Gamma(c-a)} \int_{0}^{1} \frac{u^{a-1}(1-u)^{c-a-1}}{\prod_{i=1}^{n}\left(1-u z_{i}\right)^{b_{i}}} \mathrm{~d} u
$$


where $\operatorname{Re}(c)>\operatorname{Re}(a)>0$ and $\left|\arg \left(1-z_{i}\right)\right|<\pi, i=1, \ldots, n$.

For further results and properties of this function the reader is referred to Exton [1], Srivastava and Karlsson [11], and Prudnikov, Brychkov and Marichev $[10$, Sec. 7.2.4].

Let $f(\cdot)$ be a continuous function and $\alpha_{i}>0, i=1, \ldots, r$. The integral

$$
D_{r}\left(\alpha_{1}, \ldots, \alpha_{r} ; f\right)=\int_{\substack{x_{1}>0, \ldots, x_{r}>0 \\ \sum_{i=1}^{r} x_{i}<1}} \ldots \prod_{i=1}^{r} x_{i}^{\alpha_{i}-1} f\left(\sum_{i=1}^{r} x_{i}\right) \prod_{i=1}^{r} \mathrm{~d} x_{i}
$$

is known as the Liouville-Dirichlet integral. Substituting $y_{i}=x_{i} / x, i=1, \ldots$, $r-1$ and $x=\sum_{i=1}^{r} x_{i}$ with the Jacobian $J\left(x_{1}, \ldots, x_{r-1}, x_{r} \rightarrow y_{1}, \ldots, y_{r-1}, x\right)=$ $x^{r-1}$ it is easy to see that

$$
D_{n}\left(\alpha_{1}, \ldots, \alpha_{r} ; f\right)=\frac{\prod_{i=1}^{r} \Gamma\left(\alpha_{i}\right)}{\Gamma\left(\sum_{i=1}^{r} \alpha_{i}\right)} \int_{0}^{1} x^{\sum_{i=1}^{r} \alpha_{i}-1} f(x) \mathrm{d} x .
$$

Further, by taking $f(x)=(1-x)^{\alpha_{r+1}}, \alpha_{r+1}>0$ in (4) and (5), the classical Dirichelet integral is evaluated as

$$
\int_{\substack{x_{1}>0, \ldots, x_{r}>0 \\ \sum_{i=1}^{r} x_{i}<1}} \ldots \prod_{i=1}^{r} u_{i}^{\alpha_{i}-1}\left(1-\sum_{i=1}^{r} x_{i}\right)^{\alpha_{r+1}-1} \prod_{i=1}^{r} \mathrm{~d} x_{i}=\frac{\prod_{i=1}^{r+1} \Gamma\left(\alpha_{i}\right)}{\Gamma\left(\sum_{i=1}^{r+1} \alpha_{i}\right)} .
$$

Furthermore, by setting $r=n-1, \alpha_{i}=a_{i}+j_{i}, i=1, \ldots, n$ where $a_{1}, \ldots, a_{n}$ are positive real numbers and $j_{1}, \ldots, j_{n}$ are non-negative integers in (6), we get

$$
\begin{aligned}
& \int_{\substack{x_{1}>0, \ldots, x_{n-1}>0 \\
\sum_{i=1}^{n-1} x_{i}<1}} \prod_{i=1}^{n-1} x_{i}^{a_{i}+j_{i}-1}\left(1-\sum_{i=1}^{n-1} x_{i}\right)^{a_{n}+j_{n}-1} \prod_{i=1}^{n-1} \mathrm{~d} x_{i} \\
& \quad=\frac{\prod_{i=1}^{n} \Gamma\left(a_{i}\right)}{\Gamma\left(\sum_{i=1}^{n} a_{i}\right)} \frac{\left(a_{1}\right)_{j_{1}} \cdots\left(a_{n}\right)_{j_{n}}}{\left(\sum_{i=1}^{n} a_{i}\right)_{j_{1}+\cdots+j_{n}}} .
\end{aligned}
$$

\section{The Dirichlet-Lauricella Type D Distribution}

The Dirichlet-Lauricella type D distribution is defined as follows. 
The random variables $U_{1}, \ldots, U_{n}$ are said to have a Dirichlet-Lauricella type $\mathrm{D}$ distribution with parameters $a_{1}, \ldots, a_{n} ; c ; d ; \theta_{1}, \ldots, \theta_{n}$, denoted by $\left(U_{1}, \ldots, U_{n}\right) \sim \operatorname{DLD}\left(a_{1}, \ldots, a_{n} ; c ; d ; \theta_{1}, \ldots, \theta_{n}\right)$, if their joint p.d.f. is given by

$$
K_{D} \frac{\prod_{i=1}^{n} u_{i}^{a_{i}-1}\left(1-\sum_{i=1}^{n} u_{i}\right)^{c-\sum_{i=1}^{n} a_{i}-1}}{\left(1-\sum_{i=1}^{n} \theta_{i} u_{i}\right)^{d}}, u_{i}>0, i=1, \ldots, n, \sum_{i=1}^{n} u_{i}<1,
$$

where $a_{1}>0, \ldots, a_{n}>0, c-a_{1}-\cdots-a_{n}>0,-1<\theta_{1}<1, \ldots,-1<\theta_{n}<1$ and $-\infty<d<\infty$. The normalizing constant $K_{D}$ in (8) is given by

$$
\begin{aligned}
K_{D}^{-1} & =\int_{\substack{u_{1}>0, \ldots, u_{n}>0 \\
\sum_{i=1}^{n} u_{i}<1}} \frac{\prod_{i=1}^{n} u_{i}^{a_{i}-1}\left(1-\sum_{i=1}^{n} u_{i}\right)^{c-\sum_{i=1}^{n} a_{i}-1}}{\left(1-\sum_{i=1}^{n} \theta_{i} u_{i}\right)^{d}} \prod_{i=1}^{n} \mathrm{~d} u_{i} \\
& =\frac{\prod_{i=1}^{n} \Gamma\left(a_{i}\right) \Gamma\left(c-\sum_{i=1}^{n} a_{i}\right)}{\Gamma(c)} F_{D}^{(n)}\left(d, a_{1}, \ldots, a_{n} ; c ; \theta_{1}, \ldots, \theta_{n}\right),
\end{aligned}
$$

where the last line has been obtained by using (3).

From (8), it is clear that for $d=0$, the Dirichlet-Lauricella type D distribution reduces a Dirichlet type 1 distribution with parameters $a_{1}, \ldots, a_{n}$ and $c-\sum_{i=1}^{n} a_{i}$.

For $n=1$, the p.d.f. in (8) simplifies to a generalized beta type 1 p.d.f. given by

$$
\frac{\Gamma(c)}{\Gamma\left(a_{1}\right) \Gamma\left(c-a_{1}\right){ }_{2} F_{1}\left(a_{1}, d ; c ; \theta_{1}\right)} \frac{u_{1}^{a_{1}-1}\left(1-u_{1}\right)^{c-a_{1}-1}}{\left(1-\theta_{1} u_{1}\right)^{d}}, 0<u_{1}<1,
$$

where $c>a_{1}>0,-1<\theta_{1}<1$ and ${ }_{2} F_{1}$ is the Gauss hypergeometric function. The generalized beta type 1 distribution defined by the above p.d.f. has been studied by Nagar and Rada-Mora [6], Nagar and Bedoya-Valencia [7], Libby and Novic [4], Pham-Gia and Duong [9].

Further, for $n=2$, the p.d.f. in (8) slides to a generalized bivariate beta type 1 p.d.f. defined by (Nadarajah and Kotz [5]),

$$
\begin{aligned}
& \frac{\Gamma(c)}{\Gamma\left(a_{1}\right) \Gamma\left(a_{2}\right) \Gamma\left(c-a_{1}-a_{2}\right) F_{1}\left(d, a_{1}, a_{2} ; c ; \theta_{1}, \theta_{2}\right)} \\
& \times \frac{u_{1}^{a_{1}-1} u_{2}^{a_{2}-1}\left(1-u_{1}-u_{2}\right)^{c-a_{1}-a_{2}-1}}{\left(1-\theta_{1} u_{1}-\theta_{2} u_{2}\right)^{d}}, u_{1}>0, u_{2}>0, u_{1}+u_{2}<1,
\end{aligned}
$$

where $a_{1}>0, a_{2}>0, c>a_{1}+a_{2},-1<\theta_{1}<1,-1<\theta_{2}<1$ and $F_{1}$ is the first hypergeometric function of Appell. 
Consider the transformation $Z_{i}=\left(1-\sum_{j=1}^{s} U_{j}\right)^{-1} U_{i}, i=s+1, \ldots, n$. Then, $u_{i}=\left(1-\sum_{j=1}^{s} u_{j}\right) z_{i}, i=s+1, \ldots, n$ with the Jacobian $J\left(u_{s+1}, \ldots, u_{n} \rightarrow\right.$ $\left.z_{s+1}, \ldots, z_{n}\right)=\left(1-\sum_{j=1}^{s} u_{j}\right)^{n-s}$. Substituting appropriately in (8), the joint density of $U_{1}, \ldots, U_{s}, Z_{s+1}, \ldots, Z_{n}$ is given by

$K_{D} \frac{\prod_{i=1}^{s} u_{i}^{a_{i}-1}\left(1-\sum_{i=1}^{s} u_{i}\right)^{c-\sum_{i=1}^{s} a_{i}-1} \prod_{i=s+1}^{n} z_{i}^{a_{i}-1}\left(1-\sum_{i=s+1}^{n} z_{i}\right)^{c-\sum_{i=1}^{n} a_{i}-1}}{\left[1-\sum_{i=1}^{s} \theta_{i} u_{i}-\left(1-\sum_{j=1}^{s} u_{j}\right) \sum_{i=s+1}^{n} \theta_{i} z_{i}\right]^{d}}$,

where $u_{i}>0, i=1, \ldots, s, \sum_{i=1}^{s} u_{i}<1, z_{i}>0, i=s+1, \cdots, n$, and $\sum_{i=s+1}^{n} z_{i}<1$.

Now, we find the marginal p.d.f. of $U_{1}, \ldots, U_{s}$ by integrating out $z_{s+1}, \ldots, z_{n}$ from the joint density of $U_{1}, \ldots, U_{s}, Z_{s+1}, \ldots, Z_{n}$ as

$$
\begin{aligned}
& K_{D} \frac{\prod_{i=1}^{s} u_{i}^{a_{i}-1}\left(1-\sum_{i=1}^{s} u_{i}\right)^{c-\sum_{i=1}^{s} a_{i}-1}}{\left(1-\sum_{i=1}^{s} \theta_{i} u_{i}\right)^{d}} \\
& \times \int_{\substack{z_{s+1}>0, \ldots, z_{n}>0 \\
\sum_{i=s+1}^{n} z_{i}<1}} \frac{\prod_{i=s+1}^{n} z_{i}^{a_{i}-1}\left(1-\sum_{i=s+1}^{n} z_{i}\right)^{c-\sum_{i=1}^{n} a_{i}-1}}{\left[1-\left(1-\sum_{j=1}^{s} u_{j}\right) \sum_{i=s+1}^{n} \theta_{i} z_{i} /\left(1-\sum_{i=1}^{s} \theta_{i} u_{i}\right)\right]^{d}} \prod_{i=s+1}^{n} \mathrm{~d} z_{i} .
\end{aligned}
$$

Now, using the integral representation of $F_{D}^{(n)}$, we derive the marginal p.d.f. of $U_{1}, \ldots, U_{s}$ as

$$
\begin{aligned}
& K_{D 1} \frac{\prod_{i=1}^{s} u_{i}^{a_{i}-1}\left(1-\sum_{i=1}^{s} u_{i}\right)^{c-\sum_{i=1}^{s} a_{i}-1}}{\left(1-\sum_{i=1}^{s} \theta_{i} u_{i}\right)^{d}} \\
& \times F_{D}^{(n-s)}\left(d, a_{s+1}, \ldots, a_{n} ; c-\sum_{i=1}^{s} a_{i} ; \frac{\theta_{s+1}\left(1-\sum_{i=1}^{s} u_{i}\right)}{1-\sum_{i=1}^{s} \theta_{i} u_{i}}, \ldots, \frac{\theta_{n}\left(1-\sum_{i=1}^{s} u_{i}\right)}{1-\sum_{i=1}^{s} \theta_{i} u_{i}}\right),
\end{aligned}
$$

where $u_{i}>0, i=1, \ldots, s, \sum_{i=1}^{s} u_{i}<1$ and

$$
K_{D 1}^{-1}=\frac{\prod_{i=1}^{s} \Gamma\left(a_{i}\right) \Gamma\left(c-\sum_{i=1}^{s} a_{i}\right)}{\Gamma(c)} F_{D}^{(n)}\left(d, a_{1}, \ldots, a_{n} ; c ; \theta_{1}, \ldots, \theta_{n}\right) .
$$

It is interesting to observe that the marginal density of $U_{1}, \ldots, U_{s}$ does not belong to the Dirichlet-Lauricella type D family of distributions and differs by an additional factor containing the Lauricella hypergeometric function $F_{D}$. 
From (11), it is straightforward to show that the marginal p.d.f. of $U_{s}$ is

$$
\begin{aligned}
K_{D 2} \frac{u_{s}^{a_{s}-1}\left(1-u_{s}\right)^{c-a_{s}-1}}{\left(1-\theta_{s} u_{s}\right)^{d_{s}}} F_{D}^{(n-1)}\left(d, a_{1}, \ldots, a_{s-1}, a_{s+1}, \ldots, a_{n} ; c-a_{s} ;\right. \\
\left.\frac{\theta_{1}\left(1-u_{s}\right)}{1-\theta_{s} u_{s}}, \ldots, \frac{\theta_{s-1}\left(1-u_{s}\right)}{1-\theta_{s} u_{s}}, \frac{\theta_{s+1}\left(1-u_{s}\right)}{1-\theta_{s} u_{s}}, \ldots, \frac{\theta_{n}\left(1-u_{s}\right)}{1-\theta_{s} u_{s}}\right),
\end{aligned}
$$

where

$$
K_{D 2}^{-1}=\frac{\Gamma\left(a_{s}\right) \Gamma\left(c-a_{s}\right)}{\Gamma(c)} F_{D}^{(n)}\left(d, a_{1}, \ldots, a_{n} ; c ; \theta_{1}, \ldots, \theta_{n}\right) .
$$

The marginal p.d.f. of $Z_{s+1}, \ldots, Z_{n}$ is given by

$$
\begin{aligned}
K_{D} & \frac{\prod_{i=s+1}^{n} z_{i}^{a_{i}-1}\left(1-\sum_{i=s+1}^{n} z_{i}\right)^{c-\sum_{i=1}^{n} a_{i}-1}}{\left(1-\sum_{i=s+1}^{n} \theta_{i} z_{i}\right)^{d}} \\
& \times \int_{\substack{u_{1}>0, \ldots, u_{s}>0 \\
\sum_{i=1}^{s} u_{i}<1}} \frac{\prod_{i=1}^{s} u_{i}^{a_{i}-1}\left(1-\sum_{i=1}^{s} u_{i}\right)^{c-\sum_{i=1}^{s} a_{i}-1}}{\left[1-\sum_{j=1}^{s}\left(\theta_{j}-\sum_{i=s+1}^{n} \theta_{i} z_{i}\right) u_{j} /\left(1-\sum_{i=s+1}^{n} \theta_{i} z_{i}\right)\right]^{d}} \prod_{i=1}^{s} \mathrm{~d} u_{i} .
\end{aligned}
$$

Now, evaluating the above integral by using (3), we get

$$
\begin{aligned}
& K_{D 3} \frac{\prod_{i=s+1}^{n} z_{i}^{a_{i}-1}\left(1-\sum_{i=s+1}^{n} z_{i}\right)^{c-\sum_{i=1}^{n} a_{i}-1}}{\left(1-\sum_{i=s+1}^{n} \theta_{i} z_{i}\right)^{d}} \\
& \times F_{D}^{(s)}\left(d, a_{1}, \ldots, a_{s} ; c ; \frac{\theta_{1}-\sum_{i=s+1}^{n} \theta_{i} z_{i}}{1-\sum_{i=s+1}^{n} \theta_{i} z_{i}}, \ldots, \frac{\theta_{s}-\sum_{i=s+1}^{n} \theta_{i} z_{i}}{1-\sum_{i=s+1}^{n} \theta_{i} z_{i}}\right),
\end{aligned}
$$

where

$$
K_{D 3}^{-1}=\frac{\prod_{i=s+1}^{n} \Gamma\left(a_{i}\right) \Gamma\left(c-\sum_{i=1}^{n} a_{i}\right)}{\Gamma\left(c-\sum_{i=1}^{s} a_{i}\right)} F_{D}^{(n)}\left(d, a_{1}, \ldots, a_{n} ; c ; \theta_{1}, \ldots, \theta_{n}\right) .
$$

It is well known that if $\left(U_{1}, \ldots, U_{n}\right) \sim \mathrm{D} 1\left(a_{1}, \ldots, a_{n} ; c-\sum_{i=1}^{n} a_{i}\right)$, then

$$
\left(\frac{U_{1}}{\sum_{i=1}^{n} U_{i}}, \ldots, \frac{U_{n-1}}{\sum_{i=1}^{n} U_{i}}\right) \sim \mathrm{D} 1\left(a_{1}, \ldots ; a_{n}\right)
$$

and the sum $\sum_{i=1}^{n} U_{i}$ follows a beta type 1 distribution with parameters $\sum_{i=1}^{n} a_{i}$ and $c-\sum_{i=1}^{n} a_{i}$. In the next theorem, we derive similar result for the DirichletLauricells type D distribution. 
Theorem 3.1. Let $\left(U_{1}, \ldots, U_{n}\right) \sim \operatorname{DLD}\left(a_{1}, \ldots, a_{n} ; c ; d ; \theta_{1}, \ldots, \theta_{n}\right)$ and define $U=\sum_{i=1}^{n} U_{i}$ and $X_{i}=U_{i} / U, i=1, \ldots, n-1$. Then, the joint p.d.f. of $X_{1}, \ldots, X_{n-1}$ is given as

$$
\begin{aligned}
& K_{D} \frac{\Gamma\left(\sum_{i=1}^{n} a_{i}\right) \Gamma\left(c-\sum_{i=1}^{n} a_{i}\right)}{\Gamma(c)} \prod_{i=1}^{n-1} x_{i}^{a_{i}-1}\left(1-\sum_{i=1}^{n-1} x_{i}\right)^{a_{n}-1} \\
& \times{ }_{2} F_{1}\left(\sum_{i=1}^{n} a_{i}, d ; c ; \sum_{i=1}^{n-1} \theta_{i} x_{i}+\theta_{n}\left(1-\sum_{i=1}^{n-1} x_{i}\right)\right)
\end{aligned}
$$

where $x_{i}>0, i=1, \ldots, n-1, \sum_{i=1}^{n-1} x_{i}<1$. Further, the p.d.f. of $U$ is derived as

$$
\begin{aligned}
& K_{D} \frac{\prod_{i=1}^{n} \Gamma\left(a_{i}\right)}{\Gamma\left(\sum_{i=1}^{n} a_{i}\right)} u^{\sum_{i=1}^{n} a_{i}-1}(1-u)^{c-\sum_{i=1}^{n} a_{i}-1} \\
& \times F_{D}^{(n)}\left(d, a_{1}, \ldots, a_{n} ; \sum_{i=1}^{n} a_{i} ; \theta_{1} u, \ldots, \theta_{n} u\right), 0<u<1 .
\end{aligned}
$$

Proof. Substituting $u_{i}=u x_{i}, i=1, \ldots, n-1$ and $u_{n}=u\left(1-\sum_{i=1}^{n-1} x_{i}\right)$ with the Jacobian $J\left(u_{1}, \ldots, u_{n} \rightarrow x_{1}, \ldots, x_{n-1}, u\right)=u^{n-1}$ in the p.d.f. of $\left(U_{1}, \ldots, U_{n}\right)$, we get the joint p.d.f. of $\left(X_{1}, \ldots, X_{n-1}\right)$ and $U$ as

$$
K_{D} \prod_{i=1}^{n-1} x_{i}^{a_{i}-1}\left(1-\sum_{i=1}^{n-1} x_{i}\right)^{a_{n}-1} \frac{u^{\sum_{i=1}^{n} a_{i}-1}(1-u)^{c-\sum_{i=1}^{n} a_{i}-1}}{\left[1-\sum_{i=1}^{n-1} \theta_{i} x_{i} u-\theta_{n} u\left(1-\sum_{i=1}^{n-1} x_{i}\right)\right]^{d}},
$$

where $x_{i}>0, i=1, \ldots, n-1, \sum_{i=1}^{n-1} x_{i}<1$ and $0<u<1$. Now, integrating $u$ in the above expression by using the integral representation of ${ }_{2} F_{1}$, we get the desired result. Further, writing

$$
\begin{aligned}
& {\left[1-\sum_{i=1}^{n-1} \theta_{i} x_{i} u-\theta_{n} u\left(1-\sum_{i=1}^{n-1} x_{i}\right)\right]^{-d}} \\
& \quad=\sum_{j_{1}, \ldots, j_{n}=0}^{\infty}(d)_{j_{1}+\cdots+j_{n}} \frac{\left(\theta_{1} x_{1} u\right)^{j_{1}} \cdots\left(\theta_{n-1} x_{n-1} u\right)^{j_{n-1}}\left[\theta_{n}\left(1-\sum_{i=1}^{n-1} x_{i}\right) u\right]^{j_{n}}}{j_{1} ! \cdots j_{n} !}
\end{aligned}
$$

the joint p.d.f. of $\left(X_{1}, \ldots, X_{n-1}\right)$ and $U$ is rewritten as

$$
K_{D} u^{\sum_{i=1}^{n} a_{i}-1}(1-u)^{c-\sum_{i=1}^{n} a_{i}-1} \sum_{j_{1}, \ldots, j_{n}=0}^{\infty}(d)_{j_{1}+\cdots+j_{n}}
$$




$$
\times \frac{\left(\theta_{1} u\right)^{j_{1}} \cdots\left(\theta_{n-1} u\right)^{j_{n-1}}\left(\theta_{n} u\right)^{j_{n}}}{j_{1} ! \cdots j_{n} !} \prod_{i=1}^{n-1} x_{i}^{a_{i}+j_{i}-1}\left(1-\sum_{i=1}^{n-1} x_{i}\right)^{a_{n}+j_{n}-1} .
$$

Now, integrating the above expression with respect to $x_{1}, \ldots, x_{n-1}$ by using (7) and summing the resulting series by applying (2), we get the desired result.

By definition, the product moments are obtained as

$$
\begin{aligned}
E\left[\prod_{i=1}^{n} u_{i}^{r_{i}}\right]= & \int_{\substack{u_{1}>0, \ldots, u_{n}>0 \\
\sum_{i=1}^{n} u_{i}<1}} \frac{\prod_{i=1}^{n} u_{i}^{a_{i}+r_{i}-1}\left(1-\sum_{i=1}^{n} u_{i}\right)^{c-\sum_{i=1}^{n} a_{i}-1}}{\left(1-\sum_{i=1}^{n} \theta_{i} u_{i}\right)^{d}} \prod_{i=1}^{n} \mathrm{~d} u_{i} \\
= & \frac{\Gamma(c) \prod_{i=1}^{n} \Gamma\left(a_{i}+r_{i}\right)}{\Gamma(c+r) \prod_{i=1}^{n} \Gamma\left(a_{i}\right)} \\
& \times \frac{F_{D}^{(n)}\left(d, a_{1}+r_{1}, \ldots, a_{n}+r_{n} ; c+r ; \theta_{1}, \ldots, \theta_{n}\right)}{F_{D}^{(n)}\left(d, a_{1}, \ldots, a_{n} ; c ; \theta_{1}, \ldots, \theta_{n}\right)},
\end{aligned}
$$

where $r=\sum_{i=1}^{n} r_{i}, \operatorname{Re}\left(a_{i}+r_{i}\right)>0, i=1, \ldots, n$ and $\operatorname{Re}(c+r)>0$. Further

$$
\begin{aligned}
E\left[\left(1-\sum_{i=1}^{n} u_{i}\right)^{h}\right]= & \int_{\substack{u_{1}>0, \ldots, u_{n}>0 \\
\sum_{i=1}^{n} u_{i}<1}} \frac{\prod_{i=1}^{n} u_{i}^{a_{i}-1}\left(1-\sum_{i=1}^{n} u_{i}\right)^{c+h-\sum_{i=1}^{n} a_{i}-1}}{\left(1-\sum_{i=1}^{n} \theta_{i} u_{i}\right)^{d}} \prod_{i=1}^{n} \mathrm{~d} u_{i} \\
= & \frac{\Gamma(c) \Gamma\left(c+h-\sum_{i=1}^{n} a_{i}\right)}{\Gamma(c+h) \Gamma\left(c-\sum_{i=1}^{n} a_{i}\right)} \\
& \times \frac{F_{D}^{(n)}\left(d, a_{1}, \ldots, a_{n} ; c+h ; \theta_{1}, \ldots, \theta_{n}\right)}{F_{D}^{(n)}\left(d, a_{1}, \ldots, a_{n} ; c ; \theta_{1}, \ldots, \theta_{n}\right)} .
\end{aligned}
$$

\section{Acknowledgments}

The research work of DKN and AG was supported by the Sistema Universitario de Investigación, Universidad de Antioquia under the project No. IN10182CE.

\section{References}

[1] Harold Exton, Multiple Hypergeometric Functions and Applications, Mathematics \& its Applications. Ellis Horwood Ltd., Chichester; Halsted Press [John Wiley \& Sons, Inc.], New York-London-Sydney (1976). 
[2] S. Kotz, N. Balakrishnan and N.L. Johnson, Continuous Multivariate Distributions, Vo. 1, Second Edition, John Wiley \& Sons, New York, USA (2000).

[3] A.K. Gupta and D.K. Nagar, Matrix Variate Distributions, Chapman \& Hall/CRC, Boca Raton, FL, USA (2000).

[4] D. L. Libby and M. R. Novic, Multivariate generalized beta distributions with applications to utility assessment, J. Educ. Statist, 7 (1982), 271-294. URL:http://dx.doi.org/10.2307/1164635

[5] S. Nadarajah and S. Kotz, The bivariate $F_{1}$-beta distribution, C. R. Math. Acad. Sci. Soc. R. Can., 27 (2005), no. 2, 58-64.

[6] Daya K. Nagar and Erika Alejandra Rada-Mora, Properties of multivariate beta distributions, Far East J. Theor. Stat., 24 (2008), 73-94.

[7] Daya K. Nagar and Danilo Bedoya-Valencia, Product and quotient of independent Gauss hypergeometric variables, Ingeniera y Ciencia, 7 (2011), no. $14,29-48$.

[8] Daya K. Nagar and Armando Gómez, A generalization of Dirichlet type 1 distribution, Int. J. Pure Appl. Math., 95 (2014).

[9] T. and Pham-Gia and Q. P. Duong, The generalized beta- and Fdistributions in statistical modelling, Math. Comput. Modelling, 12 (1989), 1613-1625. URL:http://dx.doi.org/10.1016/0895-7177(89)90337-3

[10] A. P. Prudnikov, Yu. A. Brychkov and O. I. Marichev, Integrals and Series. Vol. 3. More special functions. Translated from the Russian by G. G. Gould, Gordon and Breach Science Publishers, New York, USA (1990).

[11] H.M. Srivastava and P.W. Karlsson, Multiple Gaussian Hypergeometric Series, John Wiley \& Sons, New York, USA (1985). 
